\title{
Self-Calibrating Doppler Global Velocimeter with Laser Frequency Modulation
}

\author{
Andreas Fischer, Florian Sell, Lars Büttner, Jürgen Czarske \\ Technische Universität Dresden, Laboratory for Measuring and Testing Techniques, Dresden, Germany \\ E-Mail: andreas.fischer2@tu-dresden.de
}

\begin{abstract}
In order to optimize the efficiency of compressors and gas turbines, flow measurements are required for investigating important flow phenomena such as the development of vortices. For this purpose, optical measurement tools were developed capturing velocity flow fields non-intrusively. However, those systems are often designed for the use in laboratory under stable environment conditions and require a high expertise. In industrial environments, high temperatures up to several $100{ }^{\circ} \mathrm{C}$ can occur e. g. at turbo compressors, which disturbs the measurement and results in significant measurement errors. In order to allow highly resolved flow measurements also in industrial environments and for non-specialists, smart systems using a self-calibration are required. For this reason, a novel self-calibrating technique is presented decreasing automatically the temperature sensitivity. The underlying fundamental idea is to measure the Doppler frequency shift of scattered light obtaining the flow velocity and to correct changes of the slope of the calibration curve implicitly during the measurement by using a frequency modulated laser. This simplifies the application and increases the accuracy. The novel concept is implemented and successfully validated by experiments.
\end{abstract}

\section{Introduction}

For validating flow simulations e. g. in turbomachinery, precise flow field measurements are required with an increasing demand regarding the measurement uncertainty. Very often, the performance of such measurement systems suffer from unknown systematic errors. In order to correct or eliminate these errors, a self-calibration procedure has to be implemented in the measurement system. The selfcalibration capability allows to calibrate the measurement system during or at least close to the measurement, which is especially useful for industrial measurements with non-laboratory conditions. For this reason, a novel self-calibrating Doppler global velocimeter with improved precision is presented for analyzing turbulent, complex flows.

Doppler global velocimetry (DGV) is an optical technique for measuring the flow velocity fields developed in 1990 [1]. It relies on evaluating the Doppler frequency shift of laser light, which is scattered on tracer particles. Using a molecular absorption cell, a change in frequency is converted to a change in intensity, which is measured by a camera. The scattered light intensity is measured by applying a beam splitter and a second camera (reference camera). With optimized DGV systems, the errors were reduced to a minimum standard deviation of about $0.5 \mathrm{~m} / \mathrm{s}[2,3]$. However, an overall measurement uncertainty of several meters per second is typically quoted for non-lab conditions [4]. For improving the long-time stability, a self-calibration device was developed for DGV systems monitoring the calibration curve during the measurement [5]. A comparison measurement using particle image velocimetry revealed good agreement within $2 \mathrm{~m} / \mathrm{s}$.

In order to reduce the measurement uncertainty further, the concept of laser frequency modulation was introduced to DGV in 1999. Such DGV systems with laser frequency modulation do not require a reference camera. As a result, errors due to image-misalignment do not occur, which otherwise can lead to $2.3 \mathrm{~m} / \mathrm{s}$ standard deviation at conventional DGV systems [6]. Using sinusoidal laser frequency modulation, an increased laser intensity, a decreased number of measurement points with decreased spatial resolution and an avalanche photo diode array for light detection, the stochastic errors were successfully reduced: A minimum standard deviation of $0.02 \mathrm{~m} / \mathrm{s}$ was measured at a calibration object and $0.04 \mathrm{~m} / \mathrm{s}$ in a nozzle flow [7]. However, unknown systematic errors up to $\pm 0.6 \mathrm{~m} / \mathrm{s}$ remain for repeated calibration measurements over $4 \mathrm{~h}$. It is assumed, that the frequency-to-intensity conversion that is the transmission curve of the molecular absorption cell drifts [8]. For reducing this disturbance, a novel frequency modulation is described that allows self-calibration.

The article is structured as follows: A short explanation of the measurement principle of DGV without and with sinusoidal laser frequency modulation is given first in section 2. Subsequently, the novel frequency modulation is explained in section 3, which allows self-calibration for a DGV system with sinusoidal laser 
frequency modulation. The concept is compared with the state-of-the art of self-calibration procedures for DGV addressing advantages and challenges. Subsequently, the implementation of the novel concept into our current DGV system with sinusoidal laser frequency modulation is described in section 4 . First measurement results are finally presented here, which illustrate the achieved performance of the measurement system. In section 5, the article is closed by summarizing the achieved progress and discussing open issues.

\section{Measurement principle of Doppler global velocimetry (DGV)}

DGV provides non-intrusive measurements of three-componential velocity fields in flows. The underlying measurement principles of the conventional DGV technique without laser frequency modulation and the newer DGV technique with sinusoidal laser frequency modulation are briefly summarized below.

\subsection{DGV without laser frequency modulation}

The fundamental principle of all DGV systems is to measure the Doppler frequency shift of scattered light, which depends on the flow velocity [1]. The schematic setup is shown in fig. 1a: The incident laser light is scattered on scattering particles. The scattered light is shifted in frequency according the flow velocity, because the particles' velocity is assumed to equal the flow velocity (no slip assumption). The given relation between the flow velocity and the Doppler frequency shows, that one component is measured for a single laser incident direction and a single observation direction. Using three different observation directions for instance, all three velocity components can be measured, but this is not considered here further. The Doppler frequency is finally measured using a frequency-to-intensity conversion of the scattered light and a camera. As is shown in fig. 1b, the observed scattered light intensity transmitted through a molecular absorption cell depends on the Doppler frequency, when the laser frequency is stabilized at the edge of an absorption line of the absorption cell. In order to eliminate the cross-sensitivity regarding the unknown scattered light intensity, the latter is measured using a beam splitter and a reference camera (see fig. 1a). For more details about such a DGV system and its measurement capabilities, it is referred to [9].
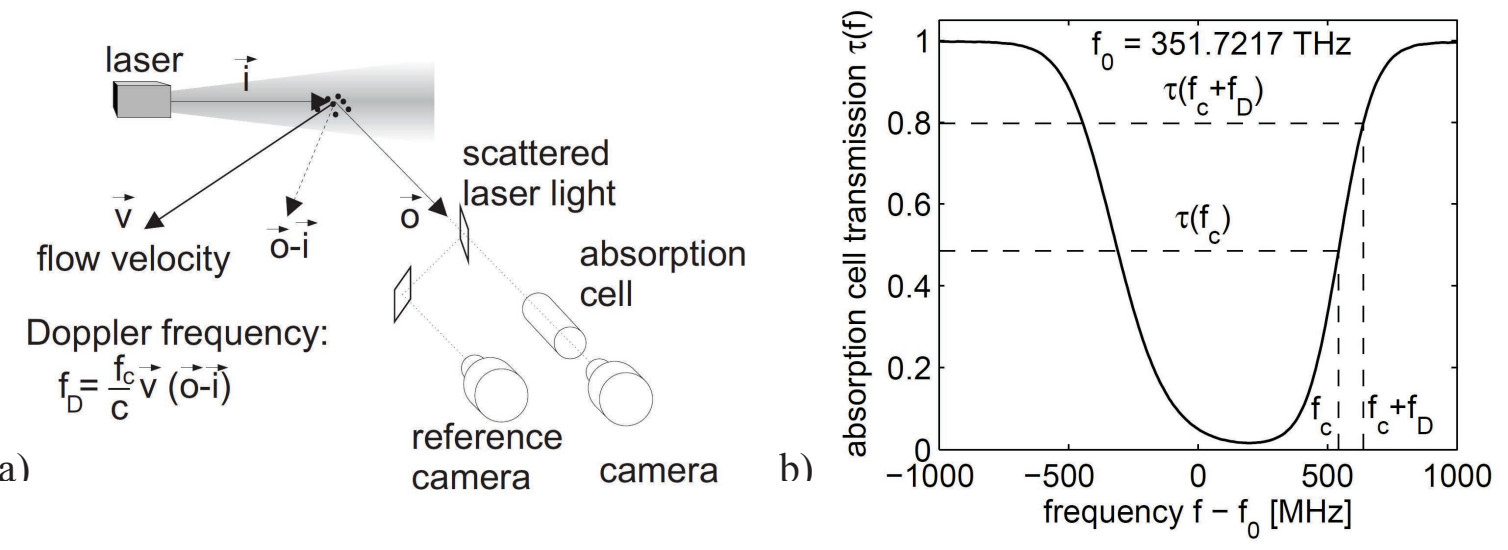

Figure 1: a) Schema of Doppler Global Velocimetry (DGV) $\left(f_{c}\right.$ represents the laser frequency and $c$ the light velocity.), b) change of transmission illustrated at a measured cesium absorption profile

\subsection{DGV with sinusoidal laser frequency modulation (FM-DGV)}

Since the usage of a reference camera can cause image misalignment errors, single camera DGV system were developed using a laser frequency modulation. The general setup of such DGV systems is shown in fig 2a. Obviously, the reference camera is omitted (cmp. fig 1a and 2a). Instead of measuring the unknown scattered light intensity directly by a reference camera, its influence is implicitly corrected by evaluating a sequence of camera images while the laser frequency is modulated. Different frequency modulation schemes can be applied either discrete frequency steps or continuous modulations [10,11].

Here, the FM-DGV approach is considered further, where a sinusoidal laser frequency modulation is used as is shown in fig. $2 \mathrm{~b}$. As a result, the scattered light signal transmitted through the absorption cell is modulated as well. The resultant signal depends on the Doppler frequency, because the laser centre frequency is stabilized and the centre frequency of the scattered light follows from the occurring Doppler effect. In order to evaluate the signal, the amplitudes $A_{1 f m}, A_{2 f m}$ of the first and second order harmonics are determined (see fig. 3a) and divided (see fig. $3 \mathrm{~b}$ ). As a result, the amplitude ratio $q=A_{1 f m} / A_{2 f m}$ is independent of the unknown scattered light power and only depends on the Doppler frequency. The relation between the quotient $q$ is shown w.r.t. the centre frequency of the scattered laser light in fig. $3 \mathrm{~b}$. 
For the absorption line at $852 \mathrm{~nm}$, the resultant measurement range corresponds to about $\pm 260 \mathrm{~m} / \mathrm{s}$ for a perpendicular arrangement of the laser incident direction and the observation direction. Within this range, the relation between the amplitude ratio and the light centre frequency is bijective.
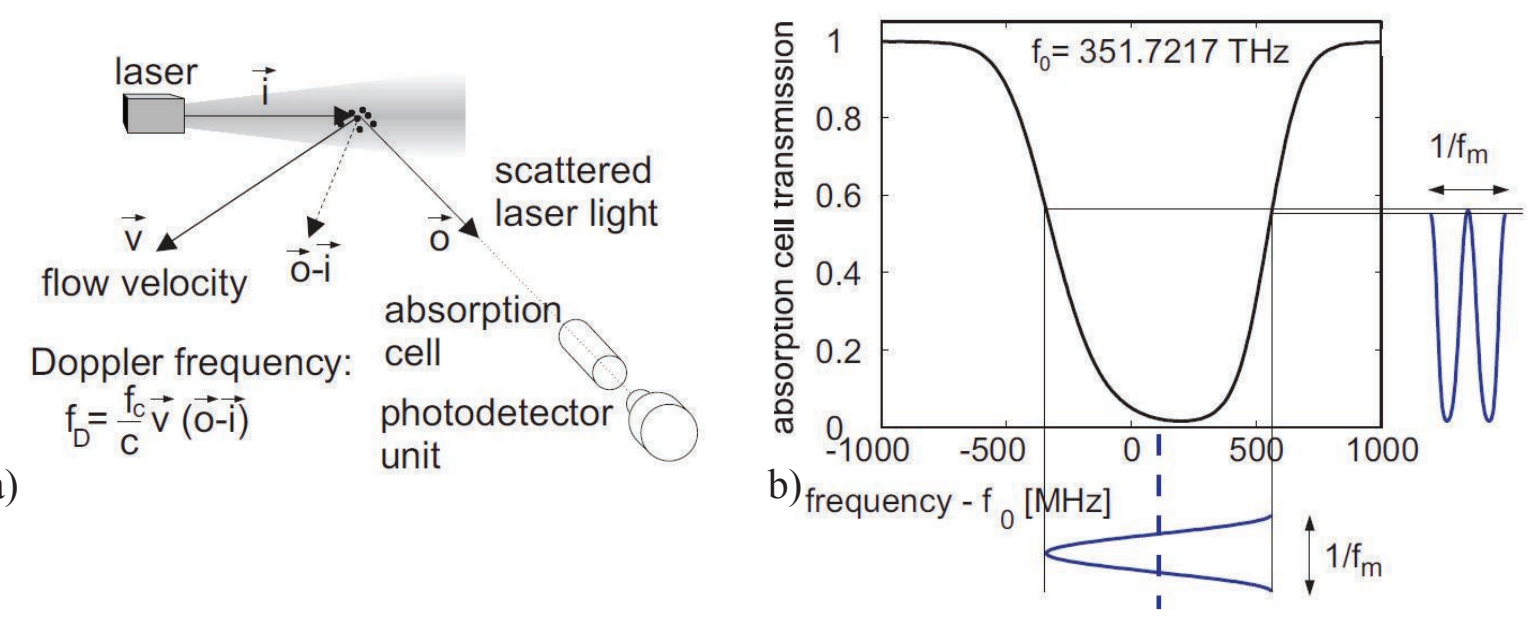

Figure: 2 a) Schema of DGV with laser frequency modulation $\left(f_{c}\right.$ represents the laser centre frequency and $c$ the light velocity.), b) measured transmission of a cesium absorption cell at near-infrared (852 $\mathrm{nm}$ ) and showing the sinusoidal laser frequency modulation for FM-DGV with the resulting transmitted light signal as an example

a)

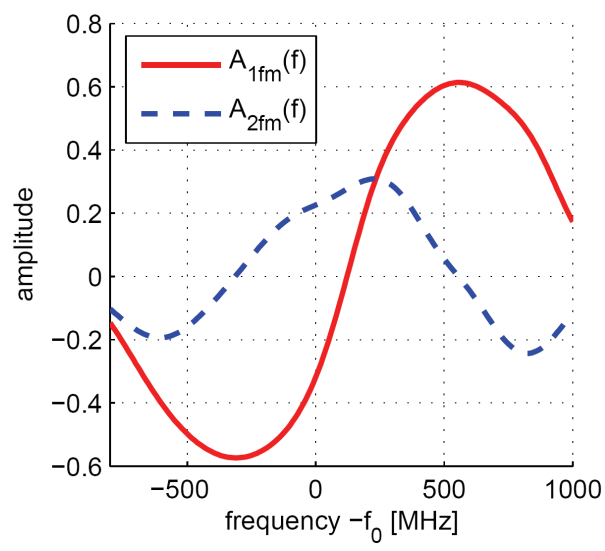

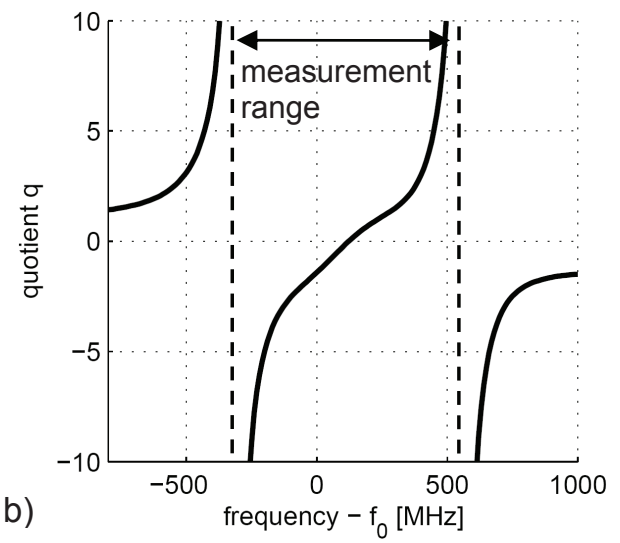

Figure 3: a) Calculated first and second order harmonic amplitudes $A_{1 f m}, A_{2 f m}$ and b) their ratio $q=A_{1 f m} / A_{2 f m}$ w.r.t. laser frequency $f_{c}+f_{D}-f_{0}$ based on the frequency modulation as illustrated in fig. $2 \mathrm{~b}$

The current FM-DGV setup consists of a diode laser $(150 \mathrm{~mW}$ max. power, narrow linewidth $<3 \mathrm{MHz}$, frequency modulation with $100 \mathrm{kHz}$ via the laser diode current), a temperature stabilized absorption cell filled with cesium gas for frequency-to-intensity conversion and a fibre-coupled avalanche photo diode array with 25 elements for fast photo detection. Since it is optimized for achieving high measurement rates up to $100 \mathrm{kHz}$, the FM-DGV system is especially capable for resolving fast time-varying flow phenomena, e. g. vortices in the wake of a cylinder, unsteady nozzle flows or vortices in turbomachine flows [12].

Although very low standard deviations down to $0.04 \mathrm{~m} / \mathrm{s}$ have been achieved e. $\mathrm{g}$. in a nozzle flow, unknown systematic errors of $\pm 0.6 \mathrm{~m} / \mathrm{s}$ limit the system performance, when investigating low speed flows. In order to overcome this limitation, a smart, self-calibrating sensor is required especially for industrial applications reducing the disturbing influence of the ambient temperature.

\section{Novel self-calibration concept using laser frequency modulation}

It is assumed, that the unknown systematic errors are mainly caused by temperature drifts of the absorption cell, which means the transmission curve is not stable. For reducing this error, a selfcalibration has to be implemented. Two principle options are known: 
a) monitoring the calibration curve during the measurement by acquiring not only scattered light, but also parts of the laser light with well-defined frequency shifts induced by acousto-optic modulators [13].

b) Applying well-defined frequency steps to the laser frequency modulation for determining the slope of the calibration curve [14].

The second option is chosen here, because it can be included in the current FM-DGV system without the requirement of adding further components. The resultant system is more compact, allows easier transportation, is cheaper and, most important, provides a calibration for each measurement channel. The concept of applying slow frequency steps has been recently demonstrated together with the usage of cameras yielding $2.5 \mathrm{~Hz}$ measurement rate [14]. In order to keep the main benefit of the current FM-DGV system achieving high measurement rates up to $100 \mathrm{kHz}$, a new modulation is required, which is more suitable for using a fast photo detector array. As a consequence, harmonic and discrete frequency modulation techniques were combined.

Both, the present and the novel laser frequency modulation is shown in fig. $4 \mathrm{a}$ and $4 \mathrm{~b}$, respectively. While the present method sinusoidally scans the non-linear absorption line from the left edge to the right edge, it is now jumped from the left edge to right edge, where the absorption line can be approximated by linear functions. At each edge, the frequency modulation is sinusoidally modulated over one period. Evaluating the amplitude ratio of the first and the second amplitude according the FM-DGV measurement principle by an harmonic analysis yields

$$
q=\frac{A_{1 f m}}{A_{2 f m}}=\frac{4}{\pi f_{h}} \cdot\left(f_{c}+f_{D}-f_{00}\right)
$$

with $f_{h}$ as amplitude of the sinusoidal modulation, $f_{c}$ as stabilized laser centre frequency, $f_{D}$ as the desired Doppler and $f_{00}$ as the solely parameter, which depends on the slope of the edges of the transmission curve and the amplitudes of the superposed rectangular and harmonic laser frequency modulation. Hence, the influence of the transmission curve can be corrected simply by measuring for zero velocity, which means measuring $q$ for the condition $f_{D}=0$. This can be accomplished before or after the measurement by inserting a resting scattering object in the measurement volume or just redirecting nonshifted laser light using mirrors into the receiving optics e. g. on-line to a separate channel or contemporary to the measurement to all channels. Here, the first option is initially implemented. The remaining slope of the calibration curve only depends on $f_{h}$, which is independent of the transmission curve and can be determined from an experiment.

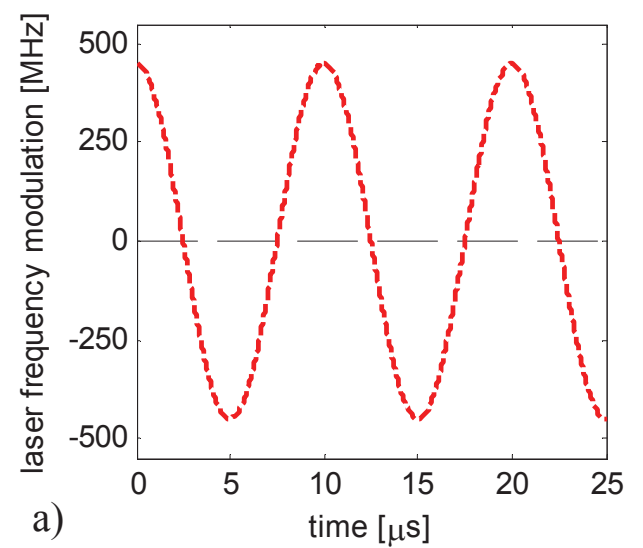

b)

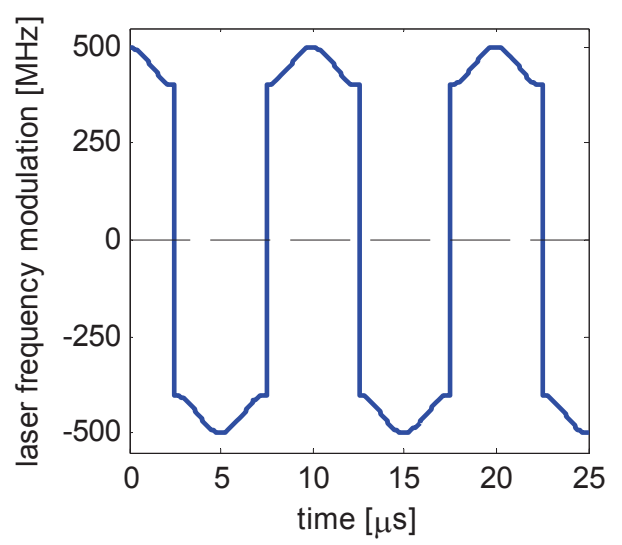

Figure 4: Calculated laser frequency modulation schemes for FM-DGV a) without (old) and b) with selfcalibration (new) combining sinusoidal and rectangular modulation

\section{Implementation and results}

In order to implement the self-calibration concept into the FM-DGV system described in section 2.2, only the arbitrary function generator, which is connected to the laser diode current control is reprogrammed. Instead of a sinusoidal modulation signal according fig. 4a, it now provides the desired modulation signal according fig. 4b. Furthermore, the modulation signal was modified accounting for the laser diode current 
to laser frequency transfer function of the laser. This is necessary for realizing the high modulation frequency of $100 \mathrm{kHz}$, which allows to achieve high measurement rates.

First, the intended reduction of the error due to temperature drifts of the absorption cell is validated by FM-DGV measurements of one channel with and without self-calibration at a rotating glass disc with rough surface usually serving as calibration object. The measurements were accomplished for different absorption cell temperatures from $20^{\circ} \mathrm{C}$ to $30^{\circ} \mathrm{C}$, where $25^{\circ} \mathrm{C}$ is the present working point. The different transmission curves are shown in fig. 5a [8]. The resultant maximum errors within the velocity range $\pm 40 \mathrm{~m} / \mathrm{s}$ are given in fig. $5 \mathrm{~b}$ w.r.t. the absorption cell temperature. The maximum velocity error for FMDGV with self-calibration is lower than $50 \%$ of the maximum error for FM-DGV without self-calibration. Hence, the error is successfully reduced by the novel self-calibration.
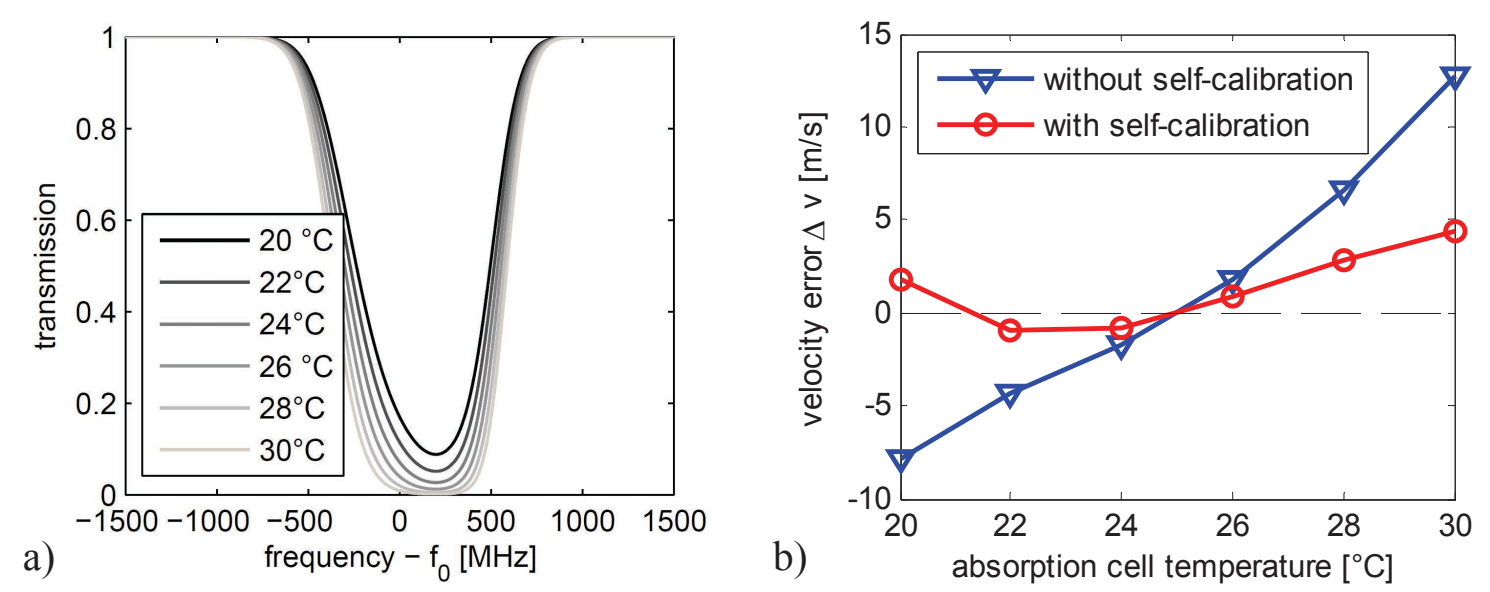

Figure 5: a) Simulated transmission curves of the cesium absorption cell for different cell temperatures (corresponding to different cesium gas densities) [8], b) maximum systematic error of velocity w.r.t. the absorption cell temperature for FM-DGV with and without self-calibration

Next, the entire systematic error of FM-DGV with and without self-calibration is considered, which is obtained from repeated measurement at the rotating glass disc each 30 min over $3.5 \mathrm{~h}$ in total. As is shown in fig. $6 a$, the error for FM-DGV without self-calibration is within $\pm 0.72 \mathrm{~m} / \mathrm{s}$ and for FM-DGV with self-calibration within $\pm 0.22 \mathrm{~m} / \mathrm{s}$. Hence, the systematic error is successfully reduced, especially for high velocities by a factor of 3 . For the sake of completeness it is also mentioned, that the stochastic errors of both FM-DGV measurements cause minimum velocity standard deviations of approximately $0.03 \mathrm{~m} / \mathrm{s}$. As an application example, the measurement of a nozzle free jet is finally shown in fig. $5 \mathrm{~b}$.
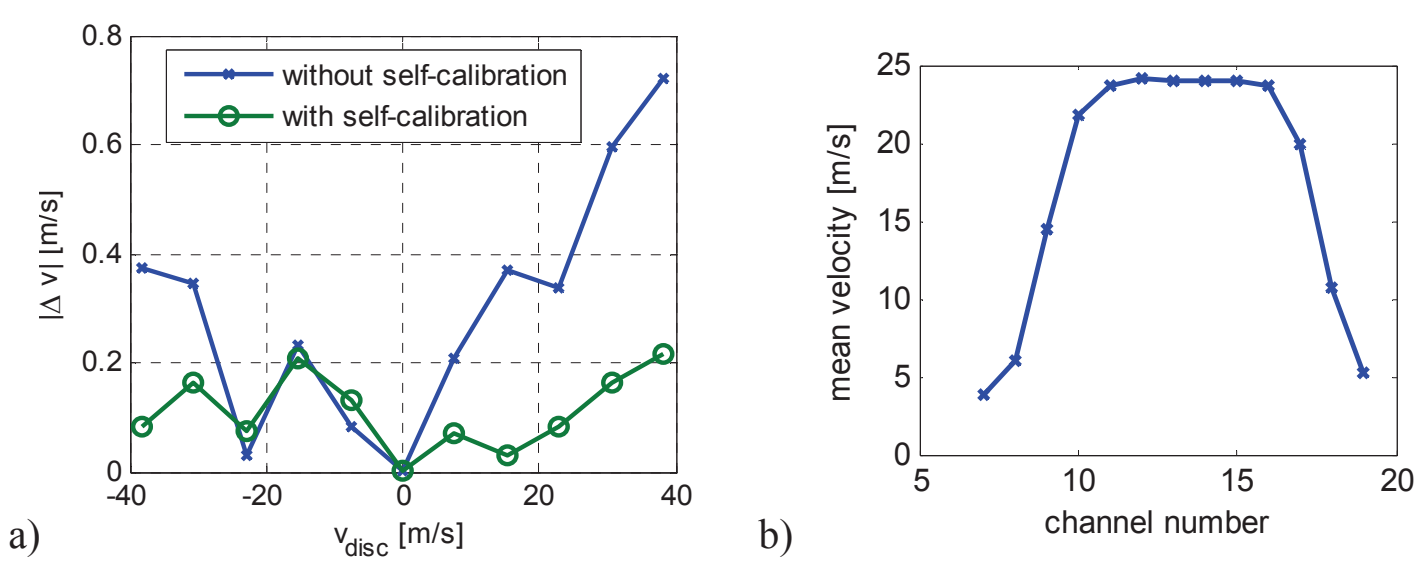

Figure 6: a) Maximum systematic velocity errors for FM-DGV with and without self-calibration determined by repeated measurement at a calibration object (rotating glass disc), b) measurement result of a nozzle free jet obtained with FM-DGV with self-calibration as an example 


\section{Summary and outlook}

A novel self-calibrating FM-DGV system was developed using a new laser frequency modulation for precise measurements of the flow velocity fields. The FM-DGV system consists of a diode laser emitting at $852 \mathrm{~nm}$ illuminating the scattering particles in the flow, a cesium absorption cell for converting the frequency shift of the scattered light due to the Doppler effect and the flow velocity to a change in intensity and a fibre-coupled avalanche photo diode array for measuring the light intensity. The new frequency modulation is a superposition of discrete and harmonic modulation, which allows to determine the Doppler frequency shift and the slope of the transmission curve edges of the absorption cell by a harmonic analysis of the detector signal. The latter feature is new and important for reducing unknown systematic errors, which currently limit the system performance. Furthermore, it simplifies the measurement reducing the calibration effort. The experiments prove a reduced cross-sensitivity for the shape of the transmission curve as intended. The error is reduced to below $50 \%$. Furthermore, the total systematic error was successfully reduced by a factor of three down to $\pm 0.22 \mathrm{~m} / \mathrm{s}$ as is validated experimentally. A further error reduction is possible by improving the quality and stability of the laser frequency modulation. As a result, the self-calibrating FM-DGV is a promising flow measurement tool for accomplishing precise velocity flow measurements also in industrial environments.

\section{Acknowledgements}

The financial support of the Deutsche Forschungsgemeinschaft (project Cz 55/22-1) is gratefully acknowledged.

\section{Literature}

[1] J. F. Meyers: Development of Doppler global velocimetry as a flow diagnostic tool, Measurement Science and Technology 6:769783, 1995.

[2] I. Röhle, I., Willert, C. E.: Extension of Doppler global velocimetry to periodic flows", Measurement Science and Technology $12: 420-431,2001$.

[3] J. F. Meyers, J. W. Lee, R. J. Schwartz: Characterization of measurement error sources in Doppler global velocimetry, Measurement Science and Technology 12:357-368, 2001.

[4] C. Willert, G. Stockhausen, J. Klinner, C. Lempereur, P. Barricau, P. Loiret, J. C. Raynal: Performance and accuracy investigations of two Doppler global velocimetry systems applied in parallel, Measurement Science and Technology 18:2504-2512, 2007.

[5] P. Barricau, C. Lempereur, J. M. Mathé, A. Mignosi, H. Buchet: Doppler Global Velocimetry: development and wind tunnel tests, in 11th International Symposium on Applications of Laser Techniques to Fluid Mechanics, No. 9.4, pp. 1-12, 2002.

[6] G. L. Morrison, C. A. Gaharan: Uncertainty estimates in DGV systems due to pixel location and velocity gradients, Measurement Science and Technology 12:369-377, 2001.

[7] A. Fischer, L. Büttner, J. Czarske, M. Eggert, G. Grosche, H. Müller: Doppler-Global-Velozimeter Investigation of time-resolved single Doppler global velocimetry using sinusoidal laser frequency modulation, Measurement Science and Technology 18: 2529$2545,2007$.

[8] A. Fischer, L. Büttner, J. Czarske, M. Eggert, H. Müller: Measurement uncertainty and temporal resolution of Doppler global velocimetry using laser frequency modulation, Applied Optics 47(21):3941-3953, 2008.

[9] R. L. McKenzie: Measurement capabilities of planar Doppler velocimetry using pulsed lasers, Applied Optics 35(6):948-964, 1996.

[10] T. O. H. Charrett, R. P. Tatam: Single camera three component planar velocity measurements using two-frequency planar Doppler velocimetry (2v-PDV), Measurement Science and Technology 17:1194-1206, 2006.

[11] H. Müller, M. Eggert, J. Czarske, L. Büttner, A. Fischer: Single-camera Doppler global velocimetry based on frequency modulation techniques, Experiments in Fluids 43:223-232, 2007.

[12] A. Fischer, L. Büttner, J. Czarske, M. Gottschall, R. Mailach, K. Vogeler: Doppler global velocimetry with laser frequency modulation for the analysis of complex turbulent flows, in 15th International Symposium on Applications of Laser Techniques to Fluid Mechanics, 1.8.2 (13 p.), Lisbon, 2010.

[13] C. Lempereur, P. Barricau, C. Gleyzes, C. Willert, G. Stockhausen, J. Klinner: Doppler Global Velocimetry in wind tunnels, Impementation issues and perfomance analysis, in 13th. Int. Symp. on Applications of Laser Techniques to Fluid Mechanics, 21.6 (12 p.), Lisbon, 2006.

[14] M. Eggert, H. Müller, J. Czarske, L. Büttner, A. Fischer: Self-calibrating Single Camera Doppler Global Velocimetry based on Frequency Shift Keying, in Imaging Measurement Methods for Flow Analysis (Eds.: W. Nitsche, C. Dobriloff), pp. 43-52, Springer, Berlin, 2009. 